\title{
Q218-public flotation jackpot
}

\section{Laura DeFrancesco}

This quarter the initial public offering (IPO) market set records for the amount of money raised while other financings dropped slightly from previous quarters. Much of the bonanza came from the East: Chinese biotechs increasingly contributed to the global picture. A notable

\section{Stock market performance}

The peak biotech stock index in 2 Q18 was within a few percentage points of its historic high from mid-2015.

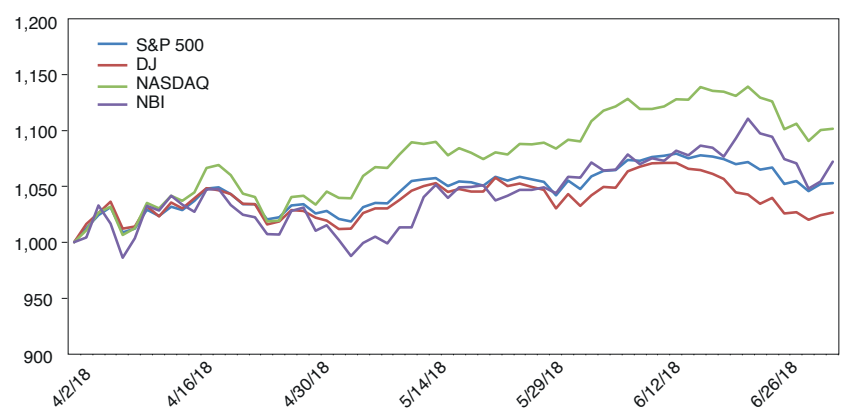

Global biotech initial public offerings

All regions showed robust IPO activity.

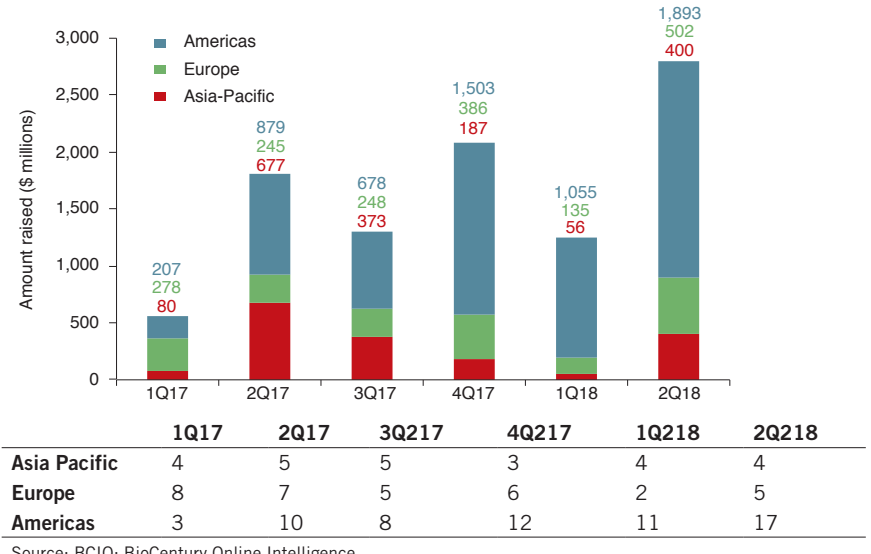

Source: $\mathrm{BCIQ}$ : BioCentury Online Intelligence.

\section{Global biotech venture capital investment}

The venture capital bonanza continues, with funds still pouring in, especially from China.

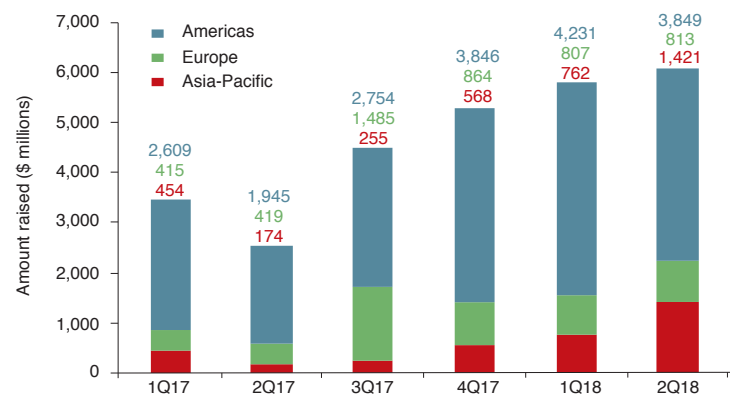

\begin{tabular}{llllllll} 
& $\mathbf{4 Q 1 6}$ & $\mathbf{1 Q 1 7}$ & $\mathbf{2 Q 1 7}$ & $\mathbf{3 Q 2 1 7}$ & $\mathbf{4 Q 2 1 7}$ & $\mathbf{1 Q 2 1 8}$ & $\mathbf{2 Q 1 8}$ \\
\hline Asia-Pacific & 10 & 10 & 11 & 8 & 14 & 15 & 20 \\
Europe & 16 & 22 & 24 & 26 & 32 & 32 & 25 \\
Americas & 51 & 85 & $\mathbf{7 3}$ & 88 & 95 & 106 & 95 \\
\hline
\end{tabular}

Source: $\mathrm{BCIQ}$ : BioCentury Online Intelligence.

Laura DeFrancesco is Senior Editor at Nature Biotechnology. number of venture capital investments (3 out of the top 10) went into precision medicine diagnostics, a sector traditionally lacking funding. Two startups developing RNA-targeted therapies were among the top 10 deals.

\section{Global biotech financing}

Last quarter saw the least debt financing and partnership money of any of the past seven quarters. ${ }^{*}$ Total deal value

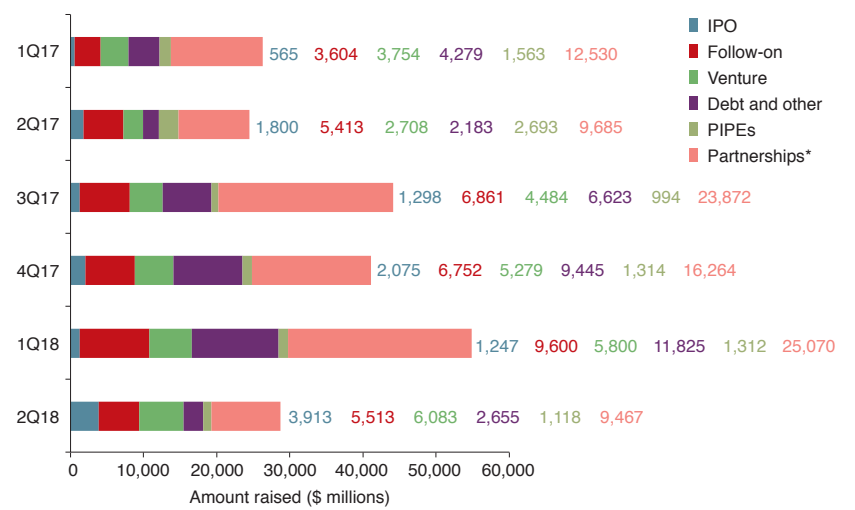

Notable 2 Q18 deals

\section{Initial public offerings}

\begin{tabular}{|c|c|c|c|}
\hline Company name & Date completed & Amount raised ( $\$$ millions) & $\begin{array}{l}\text { Change in stock since } \\
\text { offer (as of } 8 / 7 / 2018 \text { ) }\end{array}$ \\
\hline WuXi AppTec & 07-May & 353.7 & 57 \\
\hline Tricida & 27-Jun & 222.3 & -8 \\
\hline Autolus Therapeutics & 22-Jun & 172.5 & 2 \\
\hline Kiniksa Pharmaceuticals & 23-May & 170.7 & -29 \\
\hline Polyphor & 15-May & 165.3 & -14 \\
\hline Eidos Therapeutics & 19-Jun & 122.2 & -35 \\
\hline Translate Bio & 27-Jun & 121.6 & -11 \\
\hline \multicolumn{4}{|c|}{ Venture capital rounds } \\
\hline Company name & Date completed & Amount raised (\$ millions) & Round number \\
\hline Allogene Therapeutics & 03-Apr & 300 & A \\
\hline Grail & 21-May & 300 & C (oversubscribed) \\
\hline CStone Pharmaceuticals & 08-May & 260 & B \\
\hline Brii Biosciences & 24-May & 260 & Not specified \\
\hline I-Mab Biopharma & 29-Jun & 220 & B \\
\hline Helix Opco & 28-Jun & 200 & B \\
\hline Innovent Biologics & 26-Apr & 150 & $\mathrm{E}$ \\
\hline Tessa Therapeutics & 12-Apr & 130 & Not specified \\
\hline \multicolumn{4}{|c|}{ Mergers and acquisitions } \\
\hline Target & Acquirer & Value ( $\$$ millions) & Date announced \\
\hline AveXis & Novartis & 8,700 & 09-Apr \\
\hline Foundation Medicine & Roche & 2,400 & 19-Jun \\
\hline Abaxis & Zoetis & 2,000 & 15-May \\
\hline Wilson Therapeutics & Alexion Pharmaceuticals & 848 & 11-Apr \\
\hline Exosome Diagnostics & Bio-Techne & 250 & 25-Jun \\
\hline BeneVir Biopharm & Johnson \& Johnson & 140 & 02-May \\
\hline AurKa Pharma & Eli Lilly & 110 & 14-May \\
\hline Edico Genome & Illumina & 100 & 15-May \\
\hline Myonexus Therapeutics & Sarepta Therapeutics & & 03-May \\
\hline
\end{tabular}

Deals

\begin{tabular}{lll}
\hline Researcher & Investor & $\begin{array}{l}\text { Upfront Deal description } \\
\text { cash }\end{array}$ \\
\hline & Shionogi & 90
\end{tabular}

\begin{tabular}{llll}
\hline $\begin{array}{l}\text { Sage } \\
\text { Therapeutics }\end{array}$ & Shionogi & 90 & $\begin{array}{l}\text { Sage grants Shionogi exclusive rights in Japan, Taiwan and South Korea to } \\
\text { SAGE-217, a next-generation GAAA positive allosteric small-molecule }\end{array}$
\end{tabular}

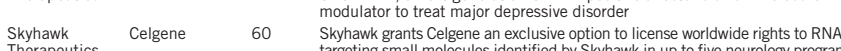
targeting small molecules identiled by Shy hawk in up to rive neurology programs Translate Bio Sanofi $\quad 45$ Translate Bio partners with Sanofi's Pasteur division to develop mRNA vacBlueprint CStone $40 \quad$ Blueprint Medicines grants CStone Pharmaceuticals exclusive rights to develop and BLU-554 in mainland China, including Hong Kong and Macau, and Taiwa Arbutus $\quad$ Roivant $\quad 38 \quad$ Arbutus Biopharma and Roivant Sciences forming JV Genevant Sciences to focus on the discovery, development and commercialization of RNA-based
therapeutics delivered via Arbutus's lipid nanoparticle and ligand conjugate

Oxford Axovant $\quad 30 \quad$ Oxford BioMedica grants Axovant exclusive, worldwide rights to develop and $\begin{array}{ll}\text { commercialize lentiviral } & \text { treat Parkinson's disease }\end{array}$

Zymeworks Daiichi Sankyo $18 \quad$ Zymeworks grants Daiichi Sankyo rights to its Azymetric and EFECT platforms develop two bispecific antibodies

$\begin{array}{lll}\text { Agios } & \text { CStone } 12 & \text { Agios Pharmaceuticals grants CStone Pharmaceuticals exclusive rights to } \\ \text { pharmaceuticals Pharmaceuticals } & \text { develop and commercialize in China (Mainland, Hong Kong, Macau and Taiwan }\end{array}$ the small molecule Tibsovo (ivosidenib), an inibitor of mulant isocitrate 